\title{
On the system of the functions $\frac{\zeta(s)}{(s-\rho)^{k}}$
}

\author{
JEAN-FRANÇOIS BURNOL
}

\begin{abstract}
The system of the functions $\frac{\zeta(s)}{(s-\beta)^{k}}$ is complete and minimal in a certain sub-Hilbert space of the $\mathrm{L}^{2}$ space of the critical line. We study whether it is also hereditarily complete.
\end{abstract}

The author thanks the CRM of Barcelona, where this work was initiated, for its hospitality, and A. Baranov, Y. Belov, and the referee for providing useful background information.

Université Lille 1

UFR de Mathématiques

Cité Scientifique M2

F-59655 Villeneuve d'Ascq

France

to be published in Complex Analysis and Operator Theory. 


\section{Introduction and statement of the main results}

The functions $\frac{\zeta(s)}{(s-\rho)^{k}}$, where $\rho$ is a non-trivial zero of the Riemann zeta function, and $\mathrm{k}$ an integer between 1 and the multiplicity $\mathrm{m}_{\rho}$ of $\rho$, are square-integrable on the critical line. In [4] I proved that they are a complete and minimal system in a certain $L \subset L^{2}\left(\frac{1}{2}+i R ; \frac{|d s|}{2 \pi}\right)$.

The Hilbert space $\mathrm{L}$ can be characterized as follows: a function $\mathrm{g}(\mathrm{s})$ belongs to $L$ if it is the Mellin transform $g(s)=\widehat{f}(s)=\int_{0}^{\infty} f(x) x^{-s} d x$ of a square integrable function $f(x)$ on $(\theta,+\infty)$, which is constant on $(\theta, 1)$ and such that its cosine transform $\int_{0}^{\infty} 2 \cos (2 \pi x y) f(y)$ dy also is constant on $(\theta, 1)$. I also proved in [4] that the dual (i.e. biorthogonal) system is complete (and minimal, of course) in L.

It is a fact that the Mellin transform $g(s)=\widehat{f}(s)$ of an $f$ satisfying these support conditions is a meromorphic function in the entire complex plane, having trivial zeros at $-2,-4, \ldots$, and at most a pole at 1 . The entire functions $s(s-1) \pi^{-\frac{s}{2}} \Gamma\left(\frac{s}{2}\right) \widehat{f}(s)$ and $s(s-1) \pi^{-\frac{s}{2}} \Gamma\left(\frac{s}{2}\right) \widehat{\mathcal{F}} f(s)$, where $\mathcal{F}$ is the Fourier cosine transform on $\mathrm{L}^{2}(\theta,+\infty ; \mathrm{dx})$, are exchanged by $\mathrm{s} \leftrightarrow 1-\mathrm{s}$. Evaluating these entire functions or their derivatives at any given $s$ defines bounded linear forms. I refer to [4] for these and other facts.

A conference talk by Yurii Belov on his joint work with Anton Baranov [1] introduced me to the notion of "hereditary completeness". Under the name of "strong completeness", it was defined by A. S. Markus about forty years ago $[6, \S 3]$ : let $\left(\mathrm{x}_{\mathrm{n}}\right)_{\mathrm{n} \in \mathrm{I}}$ be a complete and minimal system in some separable Hilbert space, and $\left(y_{n}\right)_{n \in I}$ its biorthogonal system (we shall also say "dual" for "biorthogonal"). Let $\mathrm{J} \subset \mathrm{I}$ and define the vectors $z_{n}$ by $z_{n}=x_{n}$ for $n \in J$ and $z_{n}=y_{n}$ for $n \notin J$. If, for all $J \subset I, Z_{J}=\left(z_{n}\right)_{n \in I}$ is a complete system, then $\left(x_{n}\right)$ is said to be hereditarily complete. Equivalently $([6, \S 3])$ the system $\left(x_{n}\right)$ is hereditarily complete if any vector $\mathrm{x}$ is in the closed linear span of the vectors $\left(\mathrm{y}_{\mathrm{n}}, \mathrm{x}\right) \mathrm{x}_{\mathrm{n}}$ (scalar products are linear in the second factor).

It follows from the first of these equivalent definitions that a complete and minimal system which is hereditarily complete has a complete biorthogonal. Markus constructed in [6] an example showing that this necessary condition is not sufficient. I. N. Dovbysh and N. K. Nikolskii proposed two simpler, and general, methods leading to such systems which are not hereditarily complete [3] .

Let us use as index set I the set of all couples $(\rho, \mathrm{k})$ with $\zeta(\rho)=$ (0) (non-trivial zero) and $1 \leqslant \mathrm{k} \leqslant \mathrm{m}_{\rho}$ and define $\mathrm{x}_{\rho, \mathrm{k}}=\frac{\zeta(\mathrm{s})}{(\mathrm{s}-\rho)^{\mathrm{k}}}$. From [4] these vectors are a complete and minimal system and the dual system is also complete. We study here whether this family is also hereditarily complete. The technique used to this aim will add some improvements to 
the methods from our previous publication [4], but our results are not complete: hopefully this will stimulate further researches.

To state the result, we need some notations. We do not consider arbitrary subsets $\Sigma$ of the index set I, but only those, which we call admissible, which are defined in the following manner: $\Sigma=\left\{(\rho, \mathrm{k}), 1 \leqslant \mathrm{k} \leqslant \mathrm{k}_{\Sigma}(\rho)\right\}$ where the function $\mathrm{k}_{\Sigma}: \rho \mapsto \mathrm{k}_{\Sigma}(\rho) \in\left\{\boldsymbol{\theta}, 1, \ldots, \mathrm{m}_{\rho}\right\}$ is otherwise arbitrary. The matrix of size $\mathrm{m}_{\rho} \times \mathrm{m}_{\rho}$ expressing the dual vectors $\mathrm{y}_{\rho}, \mathrm{m}_{\rho}, \mathrm{y}_{\rho}, \mathrm{m}_{\rho}-1, \ldots$, $\mathrm{y}_{\rho, 1}$ in terms of the evaluators $\mathrm{g} \mapsto \mathrm{g}(\rho), \mathrm{g} \mapsto \mathrm{g}^{\prime}(\rho), \ldots, \mathrm{g} \mapsto \mathrm{g}^{\left(\mathrm{m}_{\rho}-1\right)}(\rho)$ is upper-triangular and invertible. ${ }^{1}$ In particular, requiring that $\mathrm{g}$ should be perpendicular to $\mathrm{y}_{\rho, \mathrm{m}_{\rho}}, \mathrm{y}_{\rho, \mathrm{m}_{\rho}-1}, \ldots, \mathrm{y}_{\rho, \mathrm{k}+1}$, is equivalent to asking that $g(\rho)=g^{\prime}(\rho)=\cdots=g^{\left(m_{\rho}-k-1\right)}(\rho)=0$ (which is satisfied in particular by the $\mathrm{k}$ vectors $\left.\mathrm{g}=\mathrm{x}_{\rho, 1}, \mathrm{x}_{\rho, 2}, \ldots, \mathrm{x}_{\rho, \mathrm{k}}\right)$.

Theorem 1. Let $\Sigma$ be an admissible subset of the index set $\mathrm{I}=\{(\rho, \mathrm{k}), 1 \leqslant$ $\left.\mathrm{k} \leqslant \mathrm{m}_{\rho}\right\}$. Let $\mathcal{Z}_{\Sigma}$ be the system of the vectors $\mathrm{x}_{\rho, \mathrm{k}}, 1 \leqslant \mathrm{k} \leqslant \mathrm{k}_{\Sigma}(\rho)$, combined with the $\mathrm{y}_{\rho, \mathrm{k}}, \mathrm{k}_{\Sigma}(\rho)<\mathrm{k} \leqslant \mathrm{m}_{\rho}$ (or equivalently with the evaluators $\mathrm{g} \mapsto$ $\left.\mathrm{g}^{(j)}(\rho), \boldsymbol{\theta} \leqslant j<\mathrm{m}_{\rho}-\mathrm{k}_{\Sigma}(\rho)\right)$. The closed linear span of $\mathcal{Z}_{\Sigma}$ has at most codimension 1 in $\mathrm{L}$.

A. Baranov and Y. Belov have studied in a general manner in [1] systems of reproducing kernels in some Hilbert spaces consisting of analytic functions, identifying classes of spaces where the biorthogonal (we also say "dual") system is always complete, and giving examples where it is not complete. They examine the question of the hereditary completeness in further work [2], and in particular inside the Paley-Wiener spaces. They have a general "codimension at most 1" Theorem in this context (and will perhaps in fact exclude, under general circumstances, the codimension 1 case). In the present paper, we don't know whether codimension 1 is a true possibility or only an indication of the weaknesses of the techniques we have employed.

To prove Theorem 1 let's assume to the contrary that there are two functions perpendicular to the vectors of the system $\mathcal{Z}_{\Sigma}$, then there is one, say $G$, non trivial, and with the additional condition $G(\theta)=\theta$. We know (see [4]) that $g(s)=G(s) / s$ also belongs to L. At each $\rho$ the function $G$, hence also $g$, has order of vanishing at least equal to $m_{\rho}-k_{\Sigma}(\rho)$. And $\mathrm{G}$ is perpendicular to the $\zeta(\mathrm{s}) /(\mathrm{s}-\rho)^{\mathrm{k}}, 1 \leqslant \mathrm{k} \leqslant \mathrm{k}_{\Sigma}(\rho)$.

According to Theorem 2 (which is stated next) the function g belongs to the closed linear span of the $\zeta(\mathrm{s}) /(\mathrm{s}-\rho)^{\mathrm{k}}, 1 \leqslant \mathrm{k} \leqslant \mathrm{m}_{\rho}-\mathrm{m}_{\rho}(\mathrm{g})$, where $\mathrm{m}_{\rho}(\mathrm{g})$ is the multiplicity of $\rho$ as a (possible) zero of $g$. But $m_{\rho}(g) \geqslant m_{\rho}-k_{\Sigma}(\rho)$,

\footnotetext{
${ }^{1}$ An explicit formula shall be given later.
} 
hence $m_{\rho}-m_{\rho}(g) \leqslant k_{\Sigma}(\rho)$. So $G$ is perpendicular to $g$ :

$$
\int_{-\infty}^{\infty} \frac{\left|G\left(\frac{1}{2}+i t\right)\right|^{2}}{\frac{1}{2}+i t} d t=0
$$

Taking the real part we obtain that $G$ vanishes identically, contradiction.

Hence it suffices to prove the following:

Theorem 2. Let $\mathrm{g} \in \mathrm{L}$ belong to the domain of multiplication by $\mathrm{s}$. Then $\mathrm{g}$ is in the closed linear span of the vectors $\frac{\zeta(\mathrm{s})}{(\mathrm{s}-\rho)^{\mathrm{k}}}, 1 \leqslant \mathrm{k} \leqslant \mathrm{m}_{\rho}-\mathrm{m}_{\rho}(\mathrm{g})$, where $m_{\rho}(g)$ is the multiplicity of $\rho$ as a zero of $g$ (

We could obtain the conclusion of Theorem 2 under weaker hypotheses on g (in particular under hypotheses which do not exclude from their scope the functions $\zeta(s) /(s-\rho)$ themselves). But this would add some technical complications, which anyhow still require some conditions to be imposed upon the function $\mathrm{g}$. The stated formulation thus suffices to our goal here.

\section{Proof of Theorem 2}

Let $\phi(\mathrm{x})$ be a smooth function on $(\theta, \infty)$ with its compact support in $\left[\frac{1}{\mathrm{e}}, \mathrm{e}\right]$, and such that $\widehat{\phi}\left(\frac{1}{2}\right)=\int_{0}^{\infty} \phi(x) x^{-\frac{1}{2}} d x=1$. We will also use $\psi(x)=\frac{1}{x} \phi\left(\frac{1}{x}\right)$, which verifies $\widehat{\psi}(\mathrm{s})=\widehat{\phi}(1-\mathrm{s})$. The Mellin transform $\theta(\mathrm{s})=\widehat{\phi}(\mathrm{s})$ is an entire function which decreases faster than any inverse power of $|s|$ when $|s| \rightarrow \infty$ in any fixed vertical strip of finite width (follows immediately from integration by parts).

Note that $\widehat{\phi}\left(\frac{1}{2}+i t\right)=\int_{-1}^{1} \omega(u) e^{-i u t} d u$, where $\omega(u)=\phi\left(e^{u}\right) e^{u / 2}$, and that $\widehat{\phi}\left(\frac{1}{2}+i \epsilon \mathrm{t}\right)=\int_{-\epsilon}^{\epsilon} \frac{1}{\epsilon} \omega\left(\frac{\mathrm{u}}{\epsilon}\right) \mathrm{e}^{-i u t} \mathrm{du}$. Let $\theta_{\epsilon}(\mathrm{s})=\theta\left(\epsilon\left(\mathrm{s}-\frac{1}{2}\right)+\frac{1}{2}\right)$. On any compact this converges uniformly to the constant function 1 as $\epsilon \rightarrow \theta$, and $\theta_{\epsilon}(\mathrm{s})$ is uniformly bounded in $s$ and $\theta<\epsilon<1$ when $s$ is restricted to a vertical strip of finite width.

Let $g \in L, g(s)=\int_{0}^{\infty} f(x) x^{-s} d x$. The function $g_{\epsilon}(s)=\theta_{\epsilon}(s) g(s)$ is the Mellin transform of the multiplicative convolution:

$$
f_{\epsilon}(x)=\int_{\exp (-\epsilon)}^{\exp (+\epsilon)} \phi_{\epsilon}(t) f\left(\frac{x}{t}\right) \frac{d t}{t}
$$

with $\phi_{\epsilon}(t) \sqrt{t}=\frac{1}{\epsilon}\left(\phi\left(t^{1 / \epsilon}\right) \sqrt{t^{1 / \epsilon}}\right)$.

A Mellin transform such as $\int_{0}^{\infty} f(x) x^{-s} d x$ can also be written for $s=$ $\frac{1}{2}+$ it as $\int_{0}^{\infty} f(x) x^{1 / 2} x^{-i t} \frac{d x}{x}$, thus exhibiting it as the additive Fourier 
transform of $u \mapsto f\left(e^{u}\right) e^{u / 2}$. From this point of view we thus know that multiplying two Mellin transforms is like the additive convolution of two functions $\alpha\left(\mathrm{e}^{\mathrm{u}}\right) \mathrm{e}^{\mathrm{u} / 2}$ and $\beta\left(\mathrm{e}^{\mathrm{u}}\right) \mathrm{e}^{\mathrm{u} / 2}$ whose result should be written as a function $\gamma\left(e^{u}\right) e^{u / 2}$, thus given by

$$
\begin{aligned}
& \gamma\left(\mathrm{e}^{\mathrm{u}}\right) \mathrm{e}^{\mathrm{u} / 2}=\int_{\mathbb{R}} \alpha\left(\mathrm{e}^{\mathrm{u}-\mathrm{v}}\right) \mathrm{e}^{(\mathrm{u}-\mathrm{v}) / 2} \beta\left(\mathrm{e}^{\mathrm{v}}\right) \mathrm{e}^{\mathrm{v} / 2} \mathrm{dv}=\int_{\mathbb{R}} \alpha\left(\mathrm{e}^{\mathrm{u}-\mathrm{v}}\right) \beta\left(\mathrm{e}^{\mathrm{v}}\right) \mathrm{e}^{\mathrm{u} / 2} \mathrm{dv} \\
& \Longrightarrow \gamma(\mathrm{x})=\int_{0}^{\infty} \beta(\mathrm{t}) \alpha\left(\frac{\mathrm{x}}{\mathrm{t}}\right) \frac{\mathrm{dt}}{\mathrm{t}}
\end{aligned}
$$

This explains the formula for the multiplicative convolution $f_{\epsilon}$.

The function $f_{\epsilon}$ is constant for $\theta<x<\exp (-\epsilon)$, and its Fourier cosine transform also: indeed $\mathcal{F}\left(f_{\epsilon}\right)$ is the multiplicative convolution of $\mathcal{F}(f)$ with $\psi_{\epsilon}(\mathrm{x})=\frac{1}{\mathrm{x}} \phi_{\epsilon}\left(\frac{1}{\mathrm{x}}\right)$ (the "Intertwining formula" of [5]).

I will need a formula for $\theta_{\epsilon}(s) g(s)$ as a Mellin transform but for large $\operatorname{Re}(\mathrm{s})$. The expression $\int_{0}^{\infty} \mathrm{f}_{\epsilon}(\mathrm{x}) \mathrm{x}^{-\mathrm{s}} \mathrm{dx}$ needs modification to give an integral which makes sense for $\operatorname{Re}(s) \geqslant 1$, because of the behavior for $x \rightarrow \theta$.

Let us first look at pointwise values of $f_{\epsilon}(x)$ :

$$
\left|f_{\epsilon}(x)\right|^{2} x \leqslant \int_{\exp (-\epsilon)}^{\exp (+\epsilon)}\left|\phi_{\epsilon}(x)\right|^{2} \mathrm{dx} \int_{0}^{\infty}|f(x)|^{2} \mathrm{dx}=\mathrm{c} \epsilon^{-1}
$$

for some constant c, so in particular for $\epsilon$ fixed, we have $f_{\epsilon}(x)=O\left(x^{-1 / 2}\right)$ as $x \rightarrow+\infty$. This shows that for any $\eta>\theta, \int_{\eta}^{\infty} f_{\epsilon}(x) x^{-s} d x$ makes sense directly as an analytic function for $\operatorname{Re}(s)>\frac{1}{2}$. Although we don't really need it, let us observe that a much better bound can be obtained for $f_{\epsilon}(x)$ as $\mathrm{x} \rightarrow+\infty$. Indeed, with $\mathcal{F}$ the Fourier cosine transform, and $\psi_{\epsilon}(\mathrm{t})=$ $\frac{1}{\mathrm{t}} \phi_{\epsilon}\left(\frac{1}{\mathrm{t}}\right)$ :

$$
\begin{aligned}
f_{\epsilon}(x) & =\int_{0}^{\infty} \frac{1}{t} \phi_{\epsilon}\left(\frac{x}{t}\right) f(t) d t=\int_{0}^{\infty} \frac{1}{x} \psi_{\epsilon}\left(\frac{t}{x}\right) f(t) d t \\
& =\int_{0}^{\infty} \mathcal{F}\left(\psi_{\epsilon}\right)(x y) \mathcal{F}(f)(y) d y \\
& =\int_{0}^{1} \mathcal{F}\left(\psi_{\epsilon}\right)(x y) \beta d y+\int_{1}^{\infty} \mathcal{F}\left(\psi_{\epsilon}\right)(x y) \mathcal{F}(f)(y) d y \\
& =-\beta \int_{1}^{\infty} \mathcal{F}\left(\psi_{\epsilon}\right)(x y) d y+\int_{1}^{\infty} \mathcal{F}\left(\psi_{\epsilon}\right)(x y) \mathcal{F}(f)(y) d y
\end{aligned}
$$

Here, $\beta$ is the constant value of $\mathcal{F}(f)$ on $(\boldsymbol{\theta}, 1)$. Now, $\mathcal{F}\left(\psi_{\epsilon}\right)$ is an even function in the Schwartz class, and it follows then by elementary arguments that $f_{\epsilon}(x)$ also has Schwartz decrease as $x \rightarrow+\infty$. This is a general phenomenon related to the support property [5, §4]. In this manner, we see that in fact $\int_{\eta}^{\infty} f_{\epsilon}(x) x^{-s} d x$ directly defines an entire function of $s$, for any $\eta>0$. 
And for $\eta \leqslant \exp (-\epsilon), f_{\epsilon}(x)$ is a constant $C(\epsilon)$ on $(\theta, \eta)$ and we can compute $\int_{0}^{\eta} C(\epsilon) x^{-s} d x$ for $\operatorname{Re}(s)<1$, do the analytic continuation and reexpress it as $-\int_{\eta}^{\infty} C(\epsilon) x^{-s} d x$ for $\operatorname{Re}(s)>1$. In the end we obtain that a valid representation of $\theta_{\epsilon}(\mathrm{s}) \mathrm{g}(\mathrm{s})$ as an absolutely convergent integral, for $\operatorname{Re}(s)>1$, is

$$
\int_{\eta}^{\infty}\left(f_{\epsilon}(x)-C(\epsilon)\right) x^{-s} d x
$$

where $\eta$ is chosen $\leqslant \exp (-\epsilon)$. The quantity $C(\epsilon)$ is also the opposite of the residue of $\theta_{\epsilon}(s) g(s)$ at $s=1$, so it is $\theta\left(\epsilon \frac{1}{2}+\frac{1}{2}\right)$ times the constant value $C(\theta)$ of $f$ on $(\theta, 1)$. We have $\lim _{\epsilon \rightarrow \theta} C(\epsilon)=C(\theta)$, and at any rate this is a bounded quantity. These remarks will serve later.

The functions $f_{\epsilon}$ converge to the original $f$ in the $L^{2}$ sense as $\epsilon \rightarrow \mathbb{Q}^{+}$, but the problem is that the $g_{\epsilon}$ do not necessarily belong to $L: f_{\epsilon}$ and $\mathcal{F}\left(f_{\epsilon}\right)$ are a priori constant only on $(\theta, \exp (-\epsilon))$. In the similar computations from my paper [4] this problem was avoided by first replacing $f$ with a function with stronger support properties, but here we can't do that, at least we do not see an obvious way to regularize the function g (making it decrease in the vertical direction) while at the same time maintaining its vanishing on a certain set of zeros.

There is an a priori (polynomial in vertical strips) upper bound on the growth of $g(s)[4, T h .4 .8]$, so $g_{\epsilon}(s)=\theta_{\epsilon}(s) g(s)$ indeed decreases faster than any inverse polynomial when we go to $\infty$ in any fixed vertical strip of finite width. This allows computing some contour integrals, with the help of the following theorem:

Proposition (from [7, IX.7.]). There is a real number A and a strictly increasing sequence $T_{n}>n$ such that $|\zeta(s)|^{-1}<|s|^{A}$ on $|\operatorname{Im}(s)|=T_{n}$, $-1 \leqslant \operatorname{Re}(s) \leqslant+2$.

Note 1. (taken verbatim from [4]) from now on an infinite sum $\sum_{\rho} a(\rho)$ (with complex numbers or functions or Hilbert space vectors a $(\rho)$ 's indexed by the non-trivial zeros of the Riemann zeta function) means

$$
\lim _{n \rightarrow \infty} \sum_{|\operatorname{Im}(\rho)|<T_{n}} a(\rho),
$$

where the limit might be, if we are dealing with functions, a pointwise almost everywhere limit, or a Hilbert space limit. When we say that the partial sums are bounded (as complex numbers, or as Hilbert space vectors) we only refer to the partial sums as written above. When we say that the series is absolutely convergent it means that we group together the contributions of the $\rho$ 's with $T_{n}<|\operatorname{Im}(\rho)|<T_{n+1}$ before evaluating 
the absolute value or Hilbert norm. When building series of residues we write sometimes things as if the zeros were all simple: this is just to make the notation easier, but no hypothesis is made in this paper on the multiplicities $m_{\rho}$, and the formula used for writing $a(\rho)$ is a symbolic representation, valid for a simple zero, of the more complicated expression which would apply in case of multiplicity.

Let us follow the method of [4, Thm. 5.2], which is to consider a contour integral with

$$
\mathrm{F}(\mathrm{s})=\frac{\mathrm{g}_{\epsilon}(\mathrm{s})}{\zeta(\mathrm{s})} \frac{\zeta(\mathrm{Z})}{\mathrm{Z}-\mathrm{s}}
$$

where $Z$ is a fixed parameter. We will mainly be interested by the Z's on the critical line, but let us take it arbitrarily at this stage (distinct from 1 and from the zeros of the Riemann zeta function). We integrate F(s) on the rectangle with boundary lines $\left|\operatorname{Re}(\mathrm{s})-\frac{1}{2}\right|=\mathrm{d},|\operatorname{Im}(\mathrm{s})|=\mathrm{T}_{\mathrm{n}}$, where $\mathrm{d}>\frac{1}{2}$ is large enough so that $\left|\operatorname{Re}(\mathrm{Z})-\frac{1}{2}\right|<\mathrm{d}$. Letting $\mathrm{n} \rightarrow \infty$ we obtain:

$$
\sum_{\rho} \frac{g_{\epsilon}(\rho)}{\zeta^{\prime}(\rho)} \frac{\zeta(Z)}{Z-\rho}-g_{\epsilon}(Z)=\frac{\zeta(Z)}{2 \pi}\left(\int_{\operatorname{Re}(s)=\frac{1}{2}+d}-\int_{\operatorname{Re}(s)=\frac{1}{2}-d} \frac{g_{\epsilon}(s)}{(Z-s) \zeta(s)}|d s|\right)
$$

Let us pause to comment on the meaning of $\frac{g_{\epsilon}(\rho)}{\zeta^{\prime}(\rho)} \frac{\zeta(Z)}{Z-\rho}$ : as explained in the Note 1 , it is a symbolic notation for

$$
\operatorname{Res}_{s=\rho} \frac{g_{\epsilon}(s)}{\zeta(s)} \frac{\zeta(Z)}{Z-s}=\sum_{0 \leqslant j<m_{\rho}} \sum_{\theta \leqslant i \leqslant j} c_{j-i}(\rho) \frac{g_{\epsilon}^{(i)}(\rho)}{i !} \frac{\zeta(Z)}{(Z-\rho)^{m_{\rho}-j}}
$$

where

$$
\frac{(s-\rho)^{m_{\rho}}}{\zeta(s)}=c_{\theta}(\rho)+c_{1}(\rho)(s-\rho)+c_{2}(\rho)(s-\rho)^{2}+\cdots
$$

The linear combination $G \mapsto \sum_{\theta \leqslant i \leqslant j} c_{j-i}(\rho) \frac{G^{(i)}(\rho)}{i !}$ of evaluators, applied to $G(s)=x_{\rho^{\prime}, j^{\prime}}(s)=\zeta(s) /\left(s-\rho^{\prime}\right)^{j^{\prime}}$ gives 1 if $\rho^{\prime}=\rho$ and $j^{\prime}=m_{\rho}-j$ and $\theta$ otherwise, as can be seen from direct calculation of $\operatorname{Res}_{s=\rho} \frac{1}{\left(s-\rho^{\prime}\right)^{j^{\prime}}(Z-s)}$; it thus represents the vector $\mathrm{y}_{\rho, \mathrm{m}_{\rho}-\mathrm{j}}$ of the dual system.

The change of variable $s \mapsto 1$ - $s$ transforms the integral on the line $\operatorname{Re}(s)=\frac{1}{2}-d$ into a similar one (where $Z$ is replaced by $1-Z$ ) on the line $\operatorname{Re}(s)=\frac{1}{2}+d:$

$$
\frac{g_{\epsilon}(1-s)}{\zeta(1-s)}=\frac{\widehat{\mathcal{F}\left(f_{\epsilon}\right)}(s)}{\zeta(s)}
$$

As we have already mentioned that $\mathcal{F}\left(f_{\epsilon}\right)$ is the multiplicative convolution of $\mathcal{F}(f)$ by $\psi_{\epsilon}$, all our future arguments and bounds for the integral 
initially already defined on the line $\operatorname{Re}(s)=\frac{1}{2}+d$ would apply similarly to the integral initially on $\operatorname{Re}(\mathrm{s})=\frac{1}{2}-\mathrm{d}$.

On the line $\operatorname{Re}(s)=\frac{1}{2}+d, \zeta(s)^{-1}$ can be replaced with the absolutely convergent expression $\sum_{\mathrm{k} \geqslant 1} \mu(\mathrm{k}) \mathrm{k}^{-\mathrm{s}}$, which allows termwise integration. Let us check that for $\epsilon \leqslant \log 2$ all the contributions with $\mathrm{k} \geqslant 2$ vanish. For this we write $g_{\epsilon}(s) k^{-s}=\int_{0}^{\infty} \frac{1}{k} f_{\epsilon}\left(\frac{x}{k}\right) x^{-s} d x$. From previous discussion we know that the correct formula when $\operatorname{Re}(s)>1$ is:

$$
\int_{\eta}^{\infty}\left(\frac{1}{k} f_{\epsilon}\left(\frac{x}{k}\right)-\frac{1}{k} C(\epsilon)\right) x^{-s} d x
$$

with some $\eta \leqslant \mathrm{k} \exp (-\epsilon)$. For $\mathrm{k} \geqslant 2$ and $\epsilon \leqslant \log 2$ we can take $\eta=1$ in this formula. We want to evaluate

$$
\frac{1}{2 \pi} \int_{\operatorname{Re}(s)=\frac{1}{2}+d} \frac{g_{\epsilon}(s) k^{-s}}{Z-s}|d s|
$$

as an application of $\mathrm{Pl}$ ancherel theorem. ${ }^{2}$ So we compute the c.c. (complex conjugate):

$$
\text { c. c. }(Z-s)=\bar{Z}-(1+2 d-s)=-(1+2 d-\bar{Z}-s)
$$

With $\mathrm{w}=1+2 \mathrm{~d}-\overline{\mathrm{Z}}$, there holds $\operatorname{Re}(\mathrm{w})>\frac{1}{2}+\mathrm{d}=\operatorname{Re}(\mathrm{s})$, so

$$
(w-s)^{-1}=\int_{0}^{1} x^{w-1-s} d x=\int_{0}^{1} x^{d-\bar{Z}} x^{-\frac{1}{2}-i \operatorname{Im}(s)} d x
$$

On the other hand:

$$
g_{\epsilon}(s) k^{-s}=\int_{\eta}^{\infty}\left(\frac{1}{k} f_{\epsilon}\left(\frac{x}{k}\right)-\frac{1}{k} C(\epsilon)\right) x^{-d} x^{-\frac{1}{2}-i \operatorname{Im}(s)} d x
$$

So, by the Plancherel formula:

$$
\frac{1}{2 \pi} \int_{\operatorname{Re}(s)=\frac{1}{2}+d} \frac{g_{\epsilon}(s) k^{-s}}{Z-s}|d s|=-\int_{\min (\eta, 1)}^{1} x^{-Z}\left(\frac{1}{k} f_{\epsilon}\left(\frac{x}{k}\right)-\frac{1}{k} C(\epsilon)\right) d x
$$

For $\mathrm{k} \geqslant 2$ (and $\epsilon \leqslant \log 2$ ) we can take $\eta=1$ and this vanishes.

So we have the representation, for each given fixed $Z$ (not 1 and not a zero of the Riemann zeta function):

$$
\sum_{\rho} \frac{g_{\epsilon}(\rho)}{\zeta^{\prime}(\rho)} \frac{\zeta(Z)}{Z-\rho}-g_{\epsilon}(Z)=\underbrace{\frac{\zeta(Z)}{2 \pi} \int_{\operatorname{Re}(s)=\frac{1}{2}+d} \frac{g_{\epsilon}(s)}{Z-s}|d s|}_{A_{\epsilon}(Z)}+B_{\epsilon}(Z)
$$

\footnotetext{
${ }^{2}$ It is also possible to shift the contour of integration to the right to show that it vanishes for $\mathrm{k} \geqslant 2$ and $\epsilon \leqslant \log 2$.
} 


$$
A_{\epsilon}(Z)=-\zeta(Z) \int_{\eta}^{1} x^{-Z}\left(f_{\epsilon}(x)-C(\epsilon)\right) d x \quad \eta=\exp (-\epsilon)
$$

The convergence of the series taken over the zeros of the Riemann zeta function (and with the meaning from the Note 1) has so far only been proven pointwise. The second half of [4, Proof of 5.2] gives, on page 80 , arguments to establish that the series of functions of $\mathrm{Z}$ indexed by the zeros of the Riemann zeta function (and their multiplicities) is an absolutely convergent one in the sense of the $\mathrm{L}^{2}$-norm (and with the meaning from the Note 1 above). We do not repeat the arguments which can be applied here identically. As a corollary the sum $A_{\epsilon}(Z)+B_{\epsilon}(Z)$ is square-integrable on the critical line, a fact which is seen directly from $\int_{\eta}^{1} x^{-Z}\left(f_{\epsilon}(x)-C(\epsilon)\right) d x=O\left(\frac{1}{1+|Z|}\right)$ for $\operatorname{Re}(Z)$ bounded, obtained by an integration by parts, as $f_{\epsilon}$ is smooth. But we would also like to examine, as this would complete the proof of Theorem 2 , if the $\mathrm{L}^{2}$-norm of $\mathrm{A}_{\epsilon}(\mathrm{Z})+\mathrm{B}_{\epsilon}(\mathrm{Z})$ goes to zero as $\epsilon \rightarrow \mathbb{Q}$; this is where we will use the hypothesis that $\mathrm{sg}(\mathrm{s})$ also belongs to $\mathrm{L}$.

As an aside, for a fixed $Z$ we can show without hypothesis that $A_{\epsilon}(Z)$ goes to zero. We already mentioned that $C(\epsilon)$ was bounded, and we estimated pointwise $\left|f_{\epsilon}(x)\right| \leqslant c(x \epsilon)^{-\frac{1}{2}}$ for some constant $c$. As we integrate over $x$ in the interval from $\mathrm{e}^{-\epsilon}$ to 1 , this gives $\mathrm{A}_{\epsilon}(\mathrm{Z})=\zeta(\mathrm{Z}) O\left(\epsilon^{\frac{1}{2}}\right)$, uniformly in $Z$ for $\operatorname{Re}(Z)$ bounded.

We now bound $A_{\epsilon}(Z)$ otherwise. As we are mainly interested in $\operatorname{Re}(Z)=\frac{1}{2}$, we will from now on take $d=1$. By the Plancherel argument, or by a shift of the line of integration towards $+\infty$ :

$$
\begin{aligned}
& \frac{1}{2 \pi} \int_{\operatorname{Re}(s)=\frac{3}{2}} \frac{g(s)}{Z-s}|d s|=0 . \\
& A_{\epsilon}(Z)=\frac{\zeta(Z)}{2 \pi} \int_{\operatorname{Re}(s)=\frac{3}{2}} \frac{g_{\epsilon}(s)-g(s)}{Z-s}|d s|
\end{aligned}
$$

Writing $\frac{1}{\mathrm{Z}-\mathrm{s}}=\frac{1}{\mathrm{Z}}+\frac{\mathrm{s}}{\mathrm{Z}(\mathrm{Z}-\mathrm{s})}$ and using Cauchy-Schwarz:

$$
\begin{aligned}
& \left|A_{\epsilon}(Z)\right| \leqslant \frac{|\zeta(Z)|}{|Z|} \int_{\operatorname{Re}(s)=\frac{3}{2}}|s|\left|g_{\epsilon}(s)-g(s)\right|\left(\frac{1}{|s|}+\frac{1}{|Z-s|}\right) \frac{|d s|}{2 \pi} \\
& \left|A_{\epsilon}(Z)\right| \leqslant \frac{|\zeta(Z)|}{|Z|} \sqrt{\int_{\operatorname{Re}(s)=\frac{3}{2}}|s|^{2}\left|g_{\epsilon}(s)-g(s)\right|^{2} \frac{|d s|}{2 \pi}}\left(\sqrt{\frac{1}{3}}+\sqrt{\frac{1}{2}}\right)
\end{aligned}
$$

The last remaining integral does not depend on $\mathrm{Z}$ but is a numerical quantity depending on $\epsilon$. It goes to zero as $\epsilon \rightarrow \mathbb{\theta}$ from the Lebesgue dominated convergence theorem. We silently used that $\mathrm{sg}(\mathrm{s})$ was square-integrable on the line $\operatorname{Re}(\mathrm{s})=\frac{3}{2}$. But this is clear as, by hypothesis, $\mathrm{sg}(\mathrm{s})=$ $\frac{\mathrm{C}}{\mathrm{s}-1}+\mathrm{k}(\mathrm{s})$ with some $\mathrm{k}$ in the Hardy-space of the half-plane $\operatorname{Re}(\mathrm{s})>\frac{1}{2}$. 
Combining the results obtained we conclude that $g(Z)$ can be arbitrarily well approximated in $\mathrm{L}^{2}$-norm by a finite linear combination of the $\zeta(Z) /(Z-\rho)^{k}$ where only those $\mathrm{k}$ between 1 and $m_{\rho}-m_{\rho}(g)$ (inclusive) appear, which is the statement of Theorem 2 .

\section{The completeness of the evaluators without Krein's theorem}

In [4] I proved that the evaluators associated with the zeros of the Riemann zeta function were complete: i.e. if an element $g$ in $L$ is such that $g(s) / \zeta(s)$ is entire, then $g$ is the zero function. I used a Theorem of Krein on entire functions in the Cartwright class.

A more elementary proof can now be given. Again with $g_{\epsilon}(s)$ being defined as $\theta\left(\epsilon\left(s-\frac{1}{2}\right)+\frac{1}{2}\right) g(s)$, in the evaluation of the contour integral built with $\frac{g_{\epsilon}(s)}{\zeta(s)} \frac{\zeta(Z)}{Z-s}$ (where $Z$ is again a parameter distinct from 1 and from the zeros of the zeta function) the only singularity is now at $s=Z$, and we obtain the formula:

$$
-g_{\epsilon}(Z)=\frac{\zeta(Z)}{2 \pi}\left(\int_{\operatorname{Re}(s)=\frac{1}{2}+d}-\int_{\operatorname{Re}(s)=\frac{1}{2}-d} \frac{g_{\epsilon}(s)}{(Z-s) \zeta(s)}|d s|\right)=A_{\epsilon}(Z)+B_{\epsilon}(Z)
$$

We can as well take $\operatorname{Re}(Z)=\frac{1}{2}$ and $d=1$. But we have argued already that for fixed $Z$ there hold (under no additional hypothesis on $g$ ) the pointwise limits $A_{\epsilon}(Z) \rightarrow \mathcal{Q}, B_{\epsilon}(Z) \rightarrow \mathcal{Q}$, for $\epsilon \rightarrow \mathbb{Q}$. This proves that $g$ is the zero function.

The same argument would show that the only functions in $\mathrm{L}$ which vanish (with at least the same multiplicities) on all but perhaps finitely many zeros of the Riemann zeta function are the finite linear combinations of the functions $\frac{\zeta(s)}{(s-\rho)^{k}}$. Indeed the sum of the residues being now finite, there is no problem with taking the limit $\epsilon \rightarrow \mathbb{0}$ to obtain a pointwise identity, which suffices for the conclusion.

This gives examples of mixed systems being complete, but I must leave open the question whether codimension 1 can really happen for some other kind of combined system. 


\section{References}

[1] A. Baranov, Yu. Belov: Systems of reproducing kernels and their biorthogonal: completeness or incompleteness? IMRN, published online January 10, 2011, 33 pages. doi:10.1093/imrn/rnq281

[2] A. Baranov, Yu. Belov: work in progress. Talk at the "CRM-IMUB Workshop on Hilbert spaces of entire functions and spectral theory of self-adjoint differential operators", 4 June 2011, Barcelona.

[3] I. N. Dovbysh, N. K. Nikolskii: Two methods for avoiding hereditary completeness Journal of Soviet Mathematics, 16:3 (1981), 1175-1179. Transl. from: Zap. Nau. Sem. Len. Otd. Mat. Inst. im. V. A. Stek. AN SSSR, 65 (1976), 183-188

[4] J.-F. Burnol: Two complete and minimal systems associated with the zeros of the Riemann zeta function. J. de Th. des Nombres de Bordeaux 16 (2004), 65-94

[5] ].-F. Burnol: Entrelacement de co-Poisson. Annales de 1'Institut Fourier, 57 no. 2 (2007), 525-602

[6] A. S. Markus: The problem of spectral synthesis for operators with point spectrum, Math USSR-Izvestija, 4 (1970), No. 3, 670-696

[7] E. C. Titchmarsh: The Theory of the Riemann-Zeta Function. 2nd ed. Clarendon Press, Oxford 1986. 